doi:10.17659/01.2017.0098

Journal of Case Reports 2017;7(4):360-363

\title{
Spontaneous Intra-Abdominal Hemorrhage from Peri-Splenic Veins in an Atraumatic Patient
}

\author{
Theadora Forster-Anderson, Rita Poon \\ Department of General Surgery, Gosford Hospital, Holden Street, Gosford, NSW, Australia, 2250.
}

\section{Corresponding Author: \\ Dr. Theadora Forster-Anderson \\ Email: theadorafa@gmail.com}

This is an Open Access article distributed under the terms of the Creative Commons Attribution License (creativecommons.org/ licenses/by/3.0).

Received Accepted

Published

June 16, 2017

October 1, 2017

November 10, 2017

\begin{abstract}
Background: Isolated splenic vein bleeding without an intra-capsular splenic hemorrhage in the absence of trauma is extremely rare and not reported widely in the literature. We aim to describe a rare case of spontaneous, atraumatic peri-splenic hemorrhage to highlight the management dilemmas associated with the condition. Case Report: A 72 year-old gentleman presented with acute onset epigastric pain and nausea. His past medical history included portal vein thrombosis secondary to thrombocytosis for which he was on aspirin, and a deep vein thrombosis (DVT) and pulmonary embolus treated with warfarin. CT scan revealed a hemo-peritoneum and hemorrhage from peri-splenic veins. After failed conservative management he underwent a laparotomy for control of the bleeding. Post-operatively he developed an extensive DVT leading to limb ischemia and subsequently passed away. Conclusion: Peri-splenic venous hemorrhage in the absence of trauma is an extremely rare occurrence and is potentially fatal.
\end{abstract}

Keywords: Portal Vein, Postoperative Hemorrhage, Pulmonary Embolism, Splenic Vein, Venous Thrombosis.

\section{Introduction}

Atraumatic and non-iatrogenic peri-splenic vessel hemorrhage is an uncommon occurrence. Blunt trauma is a well-recognised cause of splenic hematomas and splenic vein hemorrhages and is responsible for the vast majority of cases. Atraumatic causes of splenic hematomas are rare in comparison and account for approximately $23 \%$ of splenic hematomas [1]. Isolated splenic vein bleeding without an intra-capsular splenic hemorrhage is far less common in the absence of trauma and not reported widely in the literature. Prompt diagnosis of peri-splenic vein bleeding is critical as the process is potentially lethal. The management of atraumatic peri-splenic vein bleeding is difficult, with limited evidence to guide treatment. We aim to describe a rare case of spontaneous, atraumatic peri-splenic hemorrhage to highlight the management dilemmas associated with this rare condition.

\section{Case Report}

A 72 year-old gentleman presented to the emergency department with acute onset of epigastric pain preceded by 24 hours of nausea. His past medical history included portal vein thrombosis secondary to essential thrombocytosis for which he was previously on aspirin, a previous epigastric hernia repair and an open prostatectomy for prostate cancer complicated by a deep vein thrombosis (DVT) and pulmonary embolus (PE). The patient developed heparin induced thrombotic thrombocytopenia syndrome after the diagnosis of the PE and was managed with an inferior vena cava filter, which was subsequently removed, and warfarin.

On presentation to the emergency department he was tachycardic, hypotensive and febrile. On examination his abdomen was generally tender but not peritonitic. A full blood 
count demonstrated an elevated white cell count of $46 \times 10^{9}$ cells per litre (normal: 4.3-10.8 $\times 10^{9}$ ), platelet count of $870 \times 10^{9}$ cells per litre (normal: $150-400 \times 10^{9}$ ); hemoglobin of $156 \mathrm{~g} / \mathrm{L}$ (normal: 140-160) and the patient's INR was 2.3 (normal: 0.81.2). A portal venous phase computed tomography (CT) scan revealed bleeding from a small region of peri-splenic veins and a hemoperitoneum. The patient stabilised with crystalloid resuscitation and warfarin reversal using pro-thrombinex. The patient was trialled for conservative management in the intensive care unit (ICU). On the advice of the hematology team, prophylactic Fondaparinux was commenced on day 2 as DVT prophylaxis and the patient was tested for clotting disorders. Platelet function assay (PFA-100) was found to be abnormal and therefore there was a presumed JAK-2 genetic abnormality, placing the patient at significantly high risk of bleeding. Further units of pro-thrombinex, fresh frozen plasma (FFP), platelets, and tranexamic acid were administered during his ICU admission. On day 3 the systolic blood pressure dropped significantly, the patient was re-scanned and taken for immediate laparotomy. A frank hemoperitoneum was encountered on entering the abdomen [Fig.1]. The abdomen was packed and the massive transfusion protocol was

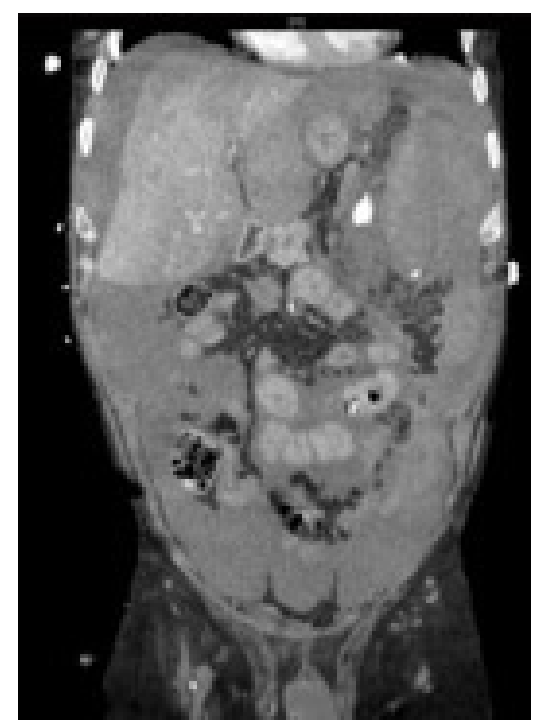

Fig.1: CT scan post second peri-splenic bleed (coronal). activated. Approximately eight litres of blood was lost during the procedure. Bleeding was found at multiple points from the peri-splenic veins, which were dilated. The hemorrhage had resulted in a large haematoma, which was mobilised off the transverse colon and splenic flexure. Attempts at suture ligation of the bleeding vessels were unsuccessful so a Medtronic EndoGIA stapler was fired across the base of the hematoma with successful arrest of active bleeding. Due to the extent of bleeding and risk of further injury intraoperatively, the decision was made to complete the procedure at this point and aim for medical optimisation. The situation was discussed with the family and the decision that the patient would not be for further surgery was made. The patient was returned to the ICU. Post-operatively he developed an extensive right lower limb DVT within the limb where his vascular access catheter had been placed. This line was removed and after consultation with the hematology and vascular teams regarding the safety of an IVC filter, the decision was made not to anti-coagulate due to the extremely high risk of peri-splenic venous re-bleeding, and therefore not to place a filter. The right lower limb DVT progressed to venous infarction and subsequent lower limb ischemia. After further discussion with the family the decision was made to palliate the patient. He passed away from multiple organ failure on day nine of admission.

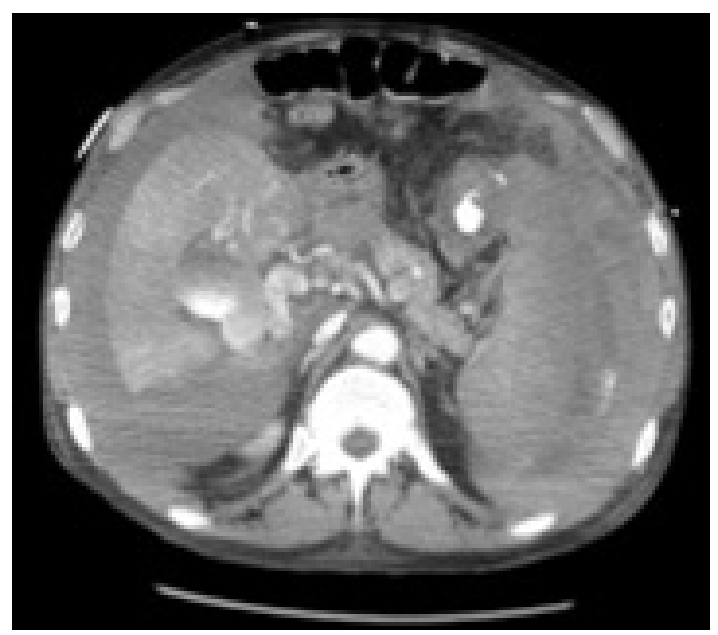

Fig.2: CT scan post second peri-splenic bleed (axial). 


\section{Discussion}

Bleeding from peri-splenic vessels in the absence of trauma is extremely rare. Spontaneous bleeding from peri-splenic vessels is usually associated with portal hypertension secondary to liver cirrhosis. Other conditions that have been implicated in atraumatic peri-splenic vessel hemorrhage or splenic rupture include hematological malignancies, infections, primary hepatomas, pancreatitis, pregnancy and amyloidosis [1]. The diagnosis of peri-splenic hemorrhage in the absence of trauma can be difficult. Blunt force trauma is commonly associated with splenic injury and is well recognised, but cases without trauma often lack the historical clues to steer the clinician to the diagnosis [1]. CT remains the most useful investigation in diagnosing splenic and perisplenic hemorrhage and often guides management $[2,3]$. The underlying pathological process leading to atraumatic hemorrhage is thought to be portal hypertension. Studies have demonstrated that in the event of portal vein thrombosis, there is vasodilation of the hepatic artery to restore adequate blood flow to the liver. There is also venous collateralisation to bypass the occluded portal vein. These compensatory mechanisms are, however, incomplete and raised portal venous pressures leads to a number of sequelae including intra-hepatic apoptosis of hepatocytes in hypoperfused areas with increased mitosis of normally perfused areas of liver parenchyma and extrahepatic variceal dilation, ascites and splenomegaly [4]. Splenomegaly is thought to further worsen the portal hypertension due to the increased splenic blood flow that accompanies the enlarged spleen [5]. The veins become dilated, friable and are more prone to spontaneous rupture. It is hypothesized that the increased splanchnic pressures further propagate bleeding when it occurs. In this patient, the process was exacerbated by the fact that the patient was on warfarin and had a pre-existing clotting disorder likely causing his initial portal vein thrombosis. The warfarin was effectively reversed with pro-thrombinex but the clotting disorder of essential thrombocytosis remained and created a delicate balance unable to be maintained in the presence of massive blood loss, blood transfusions, and the need for interventions even as simple as vascular access catheters. The decision to trial conservative management and not to proceed to immediate laparotomy was based on the positive hemodynamic response to initial resuscitation and that the patient's blood clotting issues required stabilisation. The role of conservative management in splenic trauma has been well studied and there has been a strong move away from immediate laparotomy in these patients [6]. There is a lack of evidence and guidelines to assist in the management of non-trauma patients. The situation of peri-splenic bleeding is also significantly less studied than that of bleeding within the capsule, as this is so much less common in isolation. It is not clear whether earlier operative intervention would have altered the outcome for this patient. This case is unusual in that the portal hypertension was secondary to portal vein thrombosis in the absence of liver cirrhosis. There are only a small number of cases of atraumatic splenic vein rupture reported in the literature and thrombocytosis leading to portal vein thrombosis and subsequent peri-splenic vein bleeding has not been previously reported.

\section{Conclusion}

Peri-splenic venous hemorrhage in the absence of trauma is an extremely rare occurrence and is potentially fatal. There is a lack of evidence surrounding the most appropriate course of management and further study is needed in this area.

Contributors: TFA: manuscript writing, literature review; RP: operating surgeon, case consultant and manuscript editing. TFA will act as guarantor. Both authors approved the final version of this manuscript.

Funding: None; Competing interests: None stated. 


\section{References}

1. Aubrey-Bassler FK, Sowers N. 613 cases of splenic rupture without risk factors or previously diagnosed disease: a systematic review. BMC Emergency Medicine. 2012;12:11.

2. Mortele KJ, Cantisani V, Brown DL, Ros PR. Spontaneous intraperitoneal hemorrhage: imaging features. Radiologic Clinics of North America. 2003;41:1183-1201.

3. Furlan A, Fakhran S, Federle MP. Spontaneous abdominal hemorrhage: causes, CT findings, and clinical implications. AJR American Journal of Roentgenology.
2009;193:1077-1087.

4. Ponziani FR, Zocco MA, Campanale C, Rinninella E, Tortora A, Maurizio LD, et al. Portal vein thrombosis: Insight into physiopathology, diagnosis, and treatment. World Journal of Gastroenterology. 2010;16:143-155.

5. Safioleas MC, Moulakakis KG. A rare cause of intraabdominal haemorrhage: spontaneous rupture of the splenic vein. Acta Chirurgica Belgica. 2006;106:237239.

6. Upadhyaya P. Conservative management of splenic trauma: history and current trends. Pediatric Surgery International. 2003;19:617-627. 